\title{
Hyperbolic Parabolic Equations with Nonlinearity of Kirchhoff-Carrier Type
}

\author{
E.BISOGNIN
}

ABSTRACT. In this work we study the existence of local solutions for the Cauchy problem of the hyperbolic-parabolic equation

$$
\left(K_{1} u^{\prime}\right)^{\prime}+K_{2} u^{\prime}+A u+M\left(t,\left|A^{\frac{1}{2}} u\right|^{2}\right) A u=f
$$

We represent by $A$ a self-adjoint, positive linear operator of a Hilbert space, $M$ is a real $C^{1}$-function with time dependence, such that $M(t, \eta) \geq 0$ for all $(t, \eta) \in[0, T] \times\left[0, \infty\left[\right.\right.$ and $K_{1}, K_{2}$ are real functions defined on $[0, T]$ satisfying the conditions, $K_{1}(t) \geq 0$ and $K_{2}(t) \geq \delta_{0}>0$.

The existence of local solution for $\left(^{*}\right)$ is proved by Diagonalization Theorem.

1991 Mathematics Subject Classification: 35K45, 35L15.

Servicio publicaciones Univ. Complutense. Madrid, 1995. 


\section{INTRODUCTION}

In this work we study the existence of local solutions to the initial value problem associated to the equation

$$
\left(K_{1} u^{\prime}\right)^{\prime}+K_{2} u^{\prime}-\Delta u-M\left(t, \int_{\Omega}|\nabla u(t, x)|^{2} d x\right) \Delta u=f
$$

where $\Omega$ is a bounded or unbounded open set of $\mathbb{R}^{\mathbf{n}}, \mathrm{M}$ is a real $C^{1}$ function with $M(t, \eta) \geq 0$ for all $(t, \eta) \in[0, T] \times\left[0, \infty\left[\right.\right.$ and $K_{1}, K_{2}$ are real functions defined on $[0, T]$, satisfying the conditions $K_{1}(t) \geq 0$ and $K_{2}(t) \geq \delta_{0}>0$.

Since $K_{1}(t) \geq 0$, on the set of $[0, T]$ where $K_{1}(t)=0$, the equation (1) degenerates into a parabolic case, therefore, the equation (1) is an equation of hyperbolic-parabolic type.

When $K_{1}=1$ and $K_{2}=0$, equation (1) is connected with nonlinear model of elastic strings cf. Kirchhoff [14] and Carrier [6], that describe small vibrations of a stretched string when we consider only vertical component for the tension and there is time dependence. In this case the temperature of the string at time $t$ is considered. The mathematical formulation of this model is

$$
\frac{\partial^{2} u}{\partial t^{2}}-M\left(t, \int_{\Omega}|\nabla u(x, t)|^{2} d x\right) \Delta u=f
$$

In this work we suppose $M$ a $C^{1}$-function in $[0, T] \times[0, \infty[$, with time dependence, such that $M(t, \eta) \geq 0$ and the temperature of the string, at time $t$, is decreasing.

Equations of hyperbolic-parabolic type has been studied by several authors motived by fluids with high speed, which is not our case, cf. for example Larkin [15]. In the linear case, with non identically vanishing initial data, we mention the work of Bensoussan-Lions-Papanicolau [3] and Lions [17]. In [26] Vragov studied the linear problem with null initial conditions when the functions $K_{1}$ and $K_{2}$ depend on $(x, t)$ for $(x, t) \in Q=\Omega \times[0, T]$. 
Nonlinear hyperbolic-parabolic problems were studied, among others, by Larkin [15], Medeiros [22], Gadzhiev [2], Bryukanov [5], Popivanov [25], Bubnov [4], Maciel [19] and Ferreira [11].

When $K_{1}=1, K_{2}=0$ and there is no time dependence, there is a large number of works connected with the equation

$$
\frac{\partial^{2} u}{\partial t^{2}}-M\left(\int_{\Omega}|\nabla u(x, t)|^{2} d x\right) \Delta u=f
$$

In [24] Pohozaev, [2] Arosio-Spagnolo, [1] Arosio-Garavaldi, the authors obtained global solutions to problems related with model (3). In [24] and [2] the initial data $\left\{u_{0}, u_{1}\right\}$ were choosen in a regular class of functions.

When $n=1$ we have the work of Dickey [8] in the case that $\Omega$ is the positive real line. His result was generalized for $\Omega=\mathbb{R}^{\mathbf{n}}$, by Menzala [23]. These two results were obtained by the method of Fourier transforms.

In [20], Matos obtained the local solution of (3) when $M(s) \geq m_{0}>$ 0 and $\Omega$ is a bounded or unbounded domain of $\mathbb{R}^{n}$, by Diagonalization Theorem. About local solutions of related problems with model (3), we can mention, among others, the work of Ebihara-Medeiros-Miranda [10], Crippa [7], Medeiros-Miranda [21] and Yamada [27].

In this work we study the model (1) in an abstract formulation, i.e., the eqution

$$
\left(K_{1} u^{\prime}\right)^{\prime}+K_{2} u^{\prime}+A u+M\left(t,\left|A^{\frac{1}{2}} u\right|^{2}\right) A u=f
$$

where $A$ is a self-adjoint positive operator in a real Hilbert space $H, A^{\frac{1}{2}}$ is the square root of $A$, by Diagonalization Theorem cf. Dixmier [9], Huet [13] and Lions-Magenes [18]. This method allows us to consider the cases in which there is compactness conditions or when there is no compactness conditions. Note that this method is abstract and powerfull and is not used with frequence in nonlinear problems. 


\section{TERMINOLOGY AND MAIN RESULT}

We use some definitions cf. Lions-Magenes [18], Huet [13]. A field of Hilbert space is by definition a mapping $\lambda \mapsto \mathcal{H}(\lambda)$, that for each real number $\lambda$ is associated to a Hilbert space $\mathcal{H}(\lambda)$. A vector field on real number $\mathbb{R}$ is a mapping $\lambda \mapsto u(\lambda)$ defined on $\mathbb{R}$ such that $u(\lambda) \in \mathcal{H}(\lambda)$.

The real vector space of all vector fields on $\mathbb{R}$ will be represented by $\mathcal{F}$ and by $\mu$ we represent a positive real measure.

A field of Hilbert spaces $\lambda \mapsto \mathcal{H}(\lambda)$ is said to be $\mu$-measurable when there exists a subspace $\mathcal{N}$ of $\mathcal{F}$ satisfying the conditions:

a) The mapping $\lambda \mapsto\|u(\lambda)\|_{\mathcal{H}(\lambda)}$ is $\mu$-measurable for all $u \in \mathcal{N}$.

b) If $u \in \mathcal{F}$ and $\lambda \mapsto\left(u(\lambda, v(\lambda))_{\mathcal{H}(\lambda)}\right.$ is $\mu$-measurable for all $v \in \mathcal{N}$, then $u \in \mathcal{N}$.

c) There exists in $\mathcal{N}$ a sequence $\left(u_{n}\right)_{n \in N}$ such that $\left(u_{n}(\lambda)\right)_{n \in N}$ is total on $\mathcal{H}(\lambda)$, for each $\lambda \in \mathbb{R}$.

The objects of $\mathcal{N}$ are called $\mu$-measurable vector fields. In the following, $\lambda \mapsto \mathcal{H}(\lambda)$, represents a $\mu$-measurable field of Hilbert spaces and all the vectors fields considered are $\mu$-measurable.

The space $\mathcal{H}_{0}=\int^{\oplus} \mathcal{H}(\lambda) d \mu(\lambda)$ is defined in the following way: a vector field $\lambda \mapsto u(\lambda)$ is in $\mathcal{H}_{0}$ if and only if

$$
\int_{\mathbf{R}}\|u(\lambda)\|_{\mathcal{\gamma}(\lambda)}^{2} d \mu(\lambda)<\infty
$$

Two vector fields that are equal a.e. in $\mathcal{H}_{0}$, relative to the measure $\mu$, will be identified. In $\mathcal{H}_{0}$ we define that scalar product

$$
(u, v)_{0}=\int_{\mathbf{R}}(u(\lambda), v(\lambda))_{\mathcal{H}(\lambda)} d \dot{\mu}(\lambda) \quad \text { for all } u, v \in \mathcal{H}_{0}
$$

With this scalar product, the vector space $\mathcal{H}_{0}$ turns out to be a Hilbert space, which is called the Hilbertian integral or measurable Hilbertian sum (cf. Lions and Magenes [15]) of the field $\lambda \mapsto \mathcal{H}(\lambda)$. 
By $\mathcal{H}_{\alpha}, \alpha \in \mathbb{R}$, we denote the Hilbert space of the vector fields $u$ such that the field $\lambda \mapsto \lambda^{\alpha} u(\lambda)$ is in $\mathcal{H}_{0}$. In $\mathcal{H}_{\alpha}$ we define the following norm

$$
|u|_{\alpha}^{2}=\left|\lambda^{\alpha} u\right|_{0}^{2}=\int_{\mathbf{R}} \lambda^{2 \alpha}\|u(\lambda)\|_{\mathcal{H}(\lambda)}^{2} d \mu(\lambda), \quad u \in \mathcal{H}_{\alpha} .
$$

The topological dual $\mathcal{H}_{\alpha}^{\prime}$ of $\mathcal{H}_{\alpha}$, is identified with the space $\mathcal{H}_{-\alpha}$ by the isomorphism $\sigma: \mathcal{H}_{\alpha}^{\prime} \rightarrow \mathcal{H}_{-\alpha}$ defined by $\sigma(f)=\lambda^{2 \alpha_{1}} u_{f}, f \in \mathcal{H}_{\alpha}^{\prime}$, where $u_{f}$ is obtained by using the Riesz representation theorem. The duality between $\mathcal{H}_{-\alpha}$ and $\mathcal{H}_{\alpha}$ is denoted by $\langle,\rangle_{-\alpha, \alpha}$.

Let $H$ be a separable Hilbert space. We represent by $($,$) and |\cdot|$, respectively, the inner product and norm in $H$. We consider in $H$ a self-adjoint operator $A$ such that $(A u, u) \geq 0$ for all $u \in D(A)$, where $D(A)$ is the domain of $A$.

Let $T>0$ be a fixed real number. We consider the following assumptions about the functions $M, K_{1}, K_{2}$ and initial data:

$$
\begin{aligned}
& M \in C^{1}([0, T] \times[0, \infty[, \mathbb{R}), \mathrm{M}(\mathrm{t}, \eta) \geq 0, \\
& \quad \forall(t, \eta) \in[0, T] \times[0,+\infty[. \\
& \frac{\partial}{\partial t} M(t, \eta) \leq 0, \forall(t, \eta) \in[0, T] \times[0,+\infty[. \\
& \left\{u_{0}, u_{1}, f\right\} \in D\left(A^{\frac{3}{4}}\right) \times D\left(A^{\frac{1}{4}}\right) \times L^{2}\left(0, T ; D\left(A^{\frac{1}{4}}\right)\right) . \\
& K_{1} \in W^{1, \infty}(0, T), K_{1}(t) \geq 0 . \\
& K_{2} \in L^{\infty}(0, T), K_{2}(t) \geq \delta_{0}>0 . \\
& \left.K_{2}(t)-\frac{1}{2}\left|K_{1}^{\prime}(t)\right| \geq \delta>0 \text { a.e. in }\right] 0, T[.
\end{aligned}
$$

The following theorem is the main result of this work. 
Theorem 2.1. Under the hypothesis (2.1)-(2.6) there exists $0<$ $T_{0} \leq T$ and $a$ vector function $u:\left[0, T_{0}\right] \rightarrow H$ satisfying the conditions

$$
\begin{gathered}
\left\{\begin{array}{l}
u \in L^{\infty}\left(0, T_{0}, D\left(A^{\frac{3}{4}}\right)\right) \\
u^{\prime} \in L^{2}\left(0, T_{0} ; D\left(A^{\frac{1}{4}}\right)\right) \\
\sqrt{K_{1}} u^{\prime} \in L^{\infty}\left(0, T_{0} ; D\left(A^{\frac{1}{4}}\right)\right)
\end{array}\right. \\
\left(K_{1} u^{\prime}\right)^{\prime}+K_{2} u^{\prime}+\left[1+M\left(\cdot,\left|A^{\frac{1}{2}} u\right|^{2}\right)\right] A u=f \text { in } L^{2}\left(0, T_{0} ; D\left(A^{\frac{1}{4}}\right)^{\prime}\right) \\
u(0)=u_{0}, \quad u^{\prime}(0)=u_{1} .
\end{gathered}
$$

We observe in this case that $A$ is not necessarily a coercive operator. Then we consider for each $\varepsilon>0$ the operator $A_{\varepsilon}=A+\varepsilon I$. Therefore, $A_{\varepsilon}$ satisfies the conditions of Diagonalization Theorem of. [9], [18] and [20]. It follows that there is a Hilbertian integral $\mathcal{H}_{0, \varepsilon}=\int^{\oplus} \mathcal{H}(\lambda) d \mu_{\varepsilon}(\lambda)$ where $\mu_{\varepsilon}$ is a positive Radon measure with support in $] \lambda_{\varepsilon}, \infty\left[, 0<\lambda_{\varepsilon}<\varepsilon\right.$, and an unitary operator $\mathcal{U}_{\varepsilon}$ from $H$ onto $\mathcal{H}_{0, \varepsilon}$, such that

$$
\mathcal{U}_{\varepsilon}\left(A_{\varepsilon}^{\alpha} u\right)=\lambda^{\alpha} \mathcal{U}_{\varepsilon}(u) \text { for all } u \in D\left(A_{\varepsilon}^{\alpha}\right)=D\left(A^{\alpha}\right), \alpha \geq 0
$$

$\mathcal{U}_{\varepsilon}$ is an isomorphism from $D\left(A_{\varepsilon}^{\alpha}\right)$ onto $\mathcal{H}_{\alpha, \varepsilon}$ where $D\left(A_{\varepsilon}^{\alpha}\right)$ and $D\left(A^{\alpha}\right)$ are equipped with the graph norm,

$$
|u|_{D\left(A_{\varepsilon}^{\alpha}\right)}^{2}=|u|^{2}+\left|A_{\varepsilon}^{\alpha} u\right|^{2} \quad \text { for all } u \in D\left(A_{\varepsilon}^{\alpha}\right) .
$$

For simplicity we will write $\mathcal{H}_{\alpha}$ instead of $\mathcal{H}_{\alpha, \varepsilon}, \alpha \in \mathbb{R}$ and observe that the norm $|\cdot|_{\alpha}$ of $\mathcal{H}_{\alpha}$ depends on $\varepsilon$. We also observe that if $\alpha \geq \beta$ then $\mathcal{H}_{\alpha} \subset \mathcal{H}_{\beta}$.

The vector function $u$ that satisfies Theorem 2.1 is obtained as the limit when $\varepsilon \rightarrow 0^{+}$of a net of functions $\left(u_{\varepsilon}\right)_{0<\varepsilon<1}, u_{\varepsilon}$ in the class (2.7) 
for each $\varepsilon>0$, and satisfying the family of coercive problems:

$$
\left\{\begin{array}{c}
\left(K_{1} u_{\varepsilon}^{\prime}\right)^{\prime}+K_{2} u_{\varepsilon}^{\prime}+\left[1+M\left(\cdot,\left|A_{\varepsilon}^{\frac{1}{2}} u_{\varepsilon}\right|^{2}\right)\right] A_{\varepsilon} u_{\varepsilon}=f \\
\text { in } L^{2}\left(0, T_{0} ; D\left(A^{\frac{1}{4}}\right)^{\prime}\right) \\
u_{\varepsilon}(0)=u_{0}, \quad u_{\varepsilon}^{\prime}(0)=u_{1} .
\end{array}\right.
$$

Applying formally the diagonalization operator $\mathcal{U}_{\varepsilon}$ to the equation (2.10), we have that formally $v_{\varepsilon}=\mathcal{U}_{\varepsilon}\left(u_{\varepsilon}\right)$, i.e. $v_{\varepsilon}(t)(\lambda)=v_{\varepsilon}(t, \lambda)=$ $\mathcal{U}_{\varepsilon}\left(u_{\varepsilon}(t)\right)(\lambda)$ satisfies the problem

$$
\left\{\begin{array}{l}
\left(K_{1} v_{\varepsilon}^{\prime}\right)^{\prime}+K_{2} v_{\varepsilon}^{\prime}+\left[1+M\left(\cdot,\left|v_{\varepsilon}\right|_{\frac{1}{2}}^{2}\right)\right] \lambda v_{\varepsilon}=g_{\varepsilon} \text { in } L^{2}\left(0, T_{0} ; \mathcal{H}_{-\frac{1}{4}}\right) \\
v_{\varepsilon}(0)=v_{0 \varepsilon}, \quad v_{\varepsilon}^{\prime}(0)=v_{1 \varepsilon}
\end{array}\right.
$$

where $v_{0 \varepsilon}=\mathcal{U}_{\varepsilon}\left(u_{0}\right), v_{1 \varepsilon}=\mathcal{U}_{\varepsilon}\left(u_{1}\right), g_{\varepsilon}=\mathcal{U}_{\varepsilon}(f)$.

The problem (2.11) is equivalent to (2.10) because $\mathcal{U}_{\varepsilon}$ is an isomorphism from $H$ onto $\mathcal{H}_{0, \varepsilon}$ then, if $v_{\varepsilon}$ satisfies (2.11) we have that $u_{\varepsilon}=\mathcal{U}_{\varepsilon}^{-1}\left(v_{\varepsilon}\right)$ satisfies $(2.10)$.

We also observe that on the set where $K_{1}(t)=0$, the equation (2.11) degenerates into a parabolic equation then, we perturbe the equation (2.11) adding the term $\xi v_{\varepsilon}^{\prime \prime}, 0<\xi \leq 1, \xi \in \mathbb{R}$, obtaining the perturbed problem:

$$
\left\{\begin{array}{c}
\left(K_{1 \xi} v_{\varepsilon \xi}^{\prime}\right)^{\prime}+K_{2} v_{\varepsilon \xi}^{\prime}+\left[1+M\left(\cdot,\left|v_{\varepsilon \xi}\right|_{\frac{1}{2}}^{2}\right)\right] \lambda v_{\varepsilon \xi}=g_{\varepsilon} \\
\text { in } L^{2}\left(0, T_{0} ; \mathcal{H}_{-\frac{1}{4}}\right) \\
v_{\varepsilon \xi}(0)=v_{0 \varepsilon}, \quad v_{\varepsilon \xi}^{\prime}(0)=v_{1 \varepsilon}
\end{array}\right.
$$

where $K_{1 \xi}(t)=K_{1}(t)+\xi$. 
The solution $v_{\varepsilon \xi}(t, \lambda)$ of the problem (2.12) is obtained as the limit of a sequence of local solutions $\left(v_{\varepsilon \xi k}\right)_{k \in \mathbf{N}}$, where $v_{\varepsilon \xi k}$ for each $k \in \mathbf{N}$ is a solutioin of a truncated problem associated to (2.12).

\section{TRUNCATED PROBLEM}

We denote by $\mathcal{H}_{0, k}, k \in \mathbb{N}$, the subspace of $\mathcal{H}_{0}$ of the fields $v(\lambda)$ such that $v(\lambda)=0 \mu$-a.e. on $\left[k,+\infty\left[\right.\right.$, and by $v_{k}$ the truncated field associated to $v$, such that

$$
v_{k}= \begin{cases}v & \mu-\text { a.e. on }] \lambda_{\varepsilon}, k\left[, 0<\lambda_{\varepsilon}<\varepsilon\right. \\ 0 & \mu-\text { a.e. on }[k,+\infty[\end{cases}
$$

The subspace $\mathcal{H}_{0, k}$ with the norm $\mathcal{H}_{0}$ is a Hitbert space and $v_{k} \in$ $\mathcal{H}_{0, k}$. We have that $v_{k} \rightarrow v$ strongly in $\mathcal{H}_{\alpha}$, and if $g_{k}$ is the truncated field associated to $g \in L^{p}\left(0, T ; \mathcal{H}_{\alpha}\right), 1 \leq p \leq \infty$, then $g_{k} \rightarrow g$ strongly in $L^{p}\left(0, T ; \mathcal{H}_{\alpha}\right)$.

The truncated problem associated to (2.12) consists of finding one field $v_{\varepsilon \xi k}:\left[0, T_{0}\right] \rightarrow \mathcal{H}_{0, k}, 0<T_{0} \leq T_{\text {, satisfying the conditions }}$

$$
\begin{gathered}
\left\{\begin{array}{c}
v_{\varepsilon \xi k} \in L^{\infty}\left(0, T_{0} ; \mathcal{H}_{0, k}\right) \\
v_{\varepsilon \xi k}^{\prime} \in L^{2}\left(0, T_{0} ; \mathcal{H}_{0, k}\right) \\
\sqrt{K_{1 \xi}} v_{\varepsilon \xi k}^{\prime} \in L^{\infty}\left(0, T_{0} ; \mathcal{H}_{0, k}\right)
\end{array}\right. \\
\left\{\begin{array}{c}
\left(K_{1 \xi} v_{\varepsilon \xi k}^{\prime}\right)^{\prime}+K_{2} v_{\varepsilon \xi k}^{\prime}+\lambda v_{\varepsilon \xi k}+M\left(\cdot,\left|v_{\varepsilon \xi k}\right|_{\frac{1}{2}}^{2}\right) \lambda v_{\varepsilon \xi k}=g_{\varepsilon k} \\
\text { in } L^{2}\left(0, T_{0} ; \mathcal{H}_{0, k}\right) \\
v_{\varepsilon \xi k}(0)=v_{0 \varepsilon k}, \quad v_{\varepsilon \xi k}^{\prime}(0)=v_{1 \varepsilon k},
\end{array}\right.
\end{gathered}
$$

where $v_{0 \varepsilon k}, v_{1 \varepsilon k}, g_{\varepsilon k}$ are the truncated fields associated to $v_{0 \varepsilon}, v_{1 \varepsilon}, g_{\varepsilon}$ respectively. 
We represent by $X_{k}$ the Banach space $L^{\infty}\left(0, T_{k} ; \mathcal{H}_{0, k}\right)$ equipped with the norm

$$
\left|v_{\varepsilon \xi k}\right|_{X_{k}}=\operatorname{ess} \sup _{0 \leq t \leq r_{k}}\left|v_{\varepsilon \xi k}(t)\right|_{\frac{1}{2}}, \quad v_{\varepsilon \xi k} \in X_{k}
$$

and we define the map $S_{k}$ by $S_{k}: X_{k} \rightarrow X_{k}, S_{k}\left(v_{\varepsilon \xi k}\right)=w_{\varepsilon \xi k}$, where $w_{\varepsilon \xi k}$ is the unique field that satisfies the conditions:

$$
\begin{aligned}
& \left\{w_{\varepsilon \xi k}, w_{\varepsilon \xi k}^{\prime}, \sqrt{K_{1 \xi}} v_{e \xi k}^{\prime}\right\} \in L^{\infty}\left(0, T_{k} ; \mathcal{H}_{0, k}\right) \times L^{2}\left(0, T_{k} ; \mathcal{H}_{0, k}\right) \times \\
& \times L^{\infty}\left(0, T_{k} ; \mathcal{H}_{0, k}\right) \\
& \left(K_{1 \xi} w_{\varepsilon \xi k}^{\prime}\right)^{\prime}+K_{2} w_{\varepsilon \xi k}^{\prime}+\lambda w_{\varepsilon \xi k}=g_{\varepsilon k}-M\left(,\left|v_{\varepsilon \xi k}\right|_{\frac{1}{2}}^{2}\right) \lambda v_{\varepsilon \xi k} \\
& \text { in } L^{2}\left(0, T_{k} ; \mathcal{H}_{0, k}\right) \\
& w_{\varepsilon \xi k}(0)=v_{0 \varepsilon k}, \quad w_{\varepsilon \xi k}^{\prime}(0)=v_{1 \varepsilon k} .
\end{aligned}
$$

Taking the scalar product in $\mathcal{H}_{0}$ of both sides of (3.4) with $2 w_{\varepsilon \xi k}^{\prime}(t)$ and integrating from 0 to $t \leq T_{k}$, we have

$$
\left\{\begin{array}{l}
\left|\sqrt{K_{1 \xi}} w_{\varepsilon \xi k}^{\prime}(t)\right|_{0}^{2}+\int_{0}^{t}\left(2 K_{2}+K_{1}^{\prime}\right)(s)\left|w_{\varepsilon \xi k}(s)\right|_{0}^{2} d s+\left|w_{\varepsilon \xi k}(t)\right|_{\frac{1}{2}}^{2} \leq \\
\leq\left|\sqrt{K_{1}(0)+\xi} v_{1 \varepsilon}\right|_{0}^{2}+\left|v_{0 \varepsilon}\right|_{\frac{1}{2}}^{2}+\left|G_{\varepsilon k}\right|_{2,0}^{2} \leq \\
\leq C_{0}\left[\left|v_{1 \varepsilon}\right|_{0}^{2}+\left|v_{0 \varepsilon}\right|_{\frac{1}{2}}^{2}+\left|G_{\varepsilon k}\right|_{2,0}^{2}\right]
\end{array}\right.
$$

where

$$
G_{\varepsilon, k}(t, \lambda)=g_{\varepsilon k}(t, \lambda)-M\left(t,\left|v_{\varepsilon \xi k}(t)\right|_{\frac{1}{2}}^{2}\right) \lambda v_{\varepsilon \xi k}(t, \lambda)
$$


and by $|\cdot|_{p, \alpha}$ we represent the norm of $L^{p}\left(0, T_{k} ; \mathcal{H}_{\alpha}\right), 0 \leq p \leq \infty, \alpha \in \mathbb{R}$. $C_{0}$ is a constant independent of $0<\varepsilon \leq 1,0<\xi \leq 1$ and $k \in \mathbb{N}$.

Let $v_{e \xi k} \in B_{a}=\left\{v_{k} \in X_{k},\left|v_{k}\right|_{X_{k}} \leq a\right\}$, then we have

$$
\left|G_{\varepsilon, k}\right|_{2,0}^{2} \leq 2|g|_{2,0}^{2}+2 C_{1 \varepsilon} k a^{2} T_{k}
$$

where

$$
C_{1 \varepsilon}=\max \left\{|M(t, \eta)|^{2} ; 0 \leq t \leq T_{k} ; 0 \leq \eta \leq a^{2}\right\}
$$

Substituting (3.7) into (3.6) then it follows that

$$
\left|S_{k}\left(v_{\varepsilon \xi k}\right)\right|_{X_{k}}^{2}=\left|w_{\varepsilon \xi k}\right|_{X_{k}}^{2} \leq E_{0 \varepsilon}+2 C_{0} C_{1 \varepsilon} a^{2} T_{k}
$$

where

$$
E_{0 \varepsilon}=C_{0}\left[\left|v_{1, \varepsilon}\right|_{0}^{2}+\left|v_{0 \varepsilon}\right|_{\frac{1}{2}}^{2}+2\left|g_{\varepsilon}\right|_{2,0}^{2}\right]
$$

If $v_{\varepsilon \xi k}, \widehat{v}_{\varepsilon \xi k} \in B_{a}$ and $z_{\varepsilon \xi k}=S\left(v_{\varepsilon \xi k}\right)-S\left(\widehat{v}_{\varepsilon \xi k}\right)$ then $z_{\varepsilon \xi k}$ satisfies the problem:

$$
\left\{\begin{array}{l}
\left(K_{1 \xi} z_{\varepsilon \xi k}^{\prime}\right)^{\prime}+K_{2} z_{\varepsilon \xi k}^{\prime}+\lambda z_{\varepsilon \xi k}=F_{\varepsilon \xi k} \text { in } L^{2}\left(0, T_{k} ; \mathcal{H}_{0, k}\right) \\
z_{\varepsilon \xi k}(0)=0, \quad z_{\varepsilon \xi k}^{\prime}(0)=0
\end{array}\right.
$$

where

$$
F_{\varepsilon \xi k}(t, \lambda)=M\left(t,\left|v_{\varepsilon \xi k}(t)\right|_{\frac{1}{2}}^{2}\right) \lambda v_{\varepsilon \xi k}(t, \lambda)-M\left(t,\left|\widehat{v}_{\varepsilon \xi k}(t)\right|_{\frac{1}{2}}^{2}\right) \lambda \widehat{v}_{\varepsilon \xi k}(t, \lambda)
$$

Taking the scalar product of both sides of (3.11) with $2 z_{\varepsilon \xi k}^{\prime}(t)$, integrating from 0 to $t \leq T_{k}$ and considering the hypothesis (2.2) we obtain 


$$
\begin{aligned}
\left|\sqrt{K_{1 \xi}} z_{\varepsilon \xi k}^{\prime}(t)\right|_{0}^{2}+ & \int_{0}^{t}\left(2 K_{2}(s)+K_{1}^{\prime}(s)\right)\left|z_{\varepsilon \xi k}^{\prime}(s)\right|_{0}^{2} d s+\left|z_{\varepsilon \xi k}(t)\right|_{\frac{1}{2}}^{2} \leq \\
& \frac{1}{\rho} \int_{0}^{T_{k}}\left|F_{\varepsilon \xi k}(t)\right|_{\mathcal{H}_{0, k}}^{2} d t+\rho \int_{0}^{t}\left|z_{\varepsilon \xi k}^{\prime}(s)\right|_{0}^{2} d s
\end{aligned}
$$

where $\rho>0$ is an arbitrary constant. we have

Using the condition $2 K_{2}(t)+K_{1}^{\prime}(t) \geq 2 \delta>0$ and choosing $\rho=\delta$

$$
\left|\sqrt{K_{1 \varepsilon}} z_{\varepsilon \xi k}^{\prime}(t)\right|_{0}^{2}+\int_{0}^{t}\left|z_{\varepsilon \xi k}^{\prime}(s)\right|_{0}^{2} d s+\left|z_{\varepsilon \xi k}(t)\right|_{1 / 2}^{2} \leq C_{3}\left|F_{\varepsilon \xi k}\right|_{2,0}^{2}
$$

where $C_{3}>0$ independs of $0<\varepsilon \leq 1,0<\xi \leq 1, k \in \mathbb{N}$ and $t \in\left[0, T_{k}\right]$.

From (3.2) results

$$
\left|F_{\varepsilon \xi k}\right|_{2,0}^{2} \leq\left[2 C_{1 \varepsilon} k+8 C_{2 \varepsilon} a^{4} k\right] T_{k}\left|v_{\varepsilon \xi k}-\widehat{v}_{\varepsilon \xi k}\right|_{X_{k}}^{2}
$$

where $C_{1 \varepsilon}$ is defined by (3.8) and

$$
C_{2 \varepsilon}=\max \left\{\|\nabla M(t, \eta)\|^{2}, 0 \leq t \leq T_{k}, 0 \leq \eta \leq 2 a^{2}\right\}
$$

Substituting (3.14) into (3.13) we obtain

$$
\begin{aligned}
& \left|\sqrt{K_{1 \xi}} z_{\varepsilon \xi k}^{\prime}(t)\right|_{0}^{2}+\int_{0}^{t}\left|z_{\varepsilon \xi k}^{\prime}(s)\right|_{0}^{2} d s+\left|z_{\varepsilon \xi k}(t)\right|_{\frac{1}{2}}^{2} \leq \\
& \leq C_{3}\left[2 C_{1 \varepsilon} k+8 C_{2 \varepsilon} a^{4} k\right] T_{k}\left|v_{\varepsilon \xi k}-\widehat{v}_{\varepsilon \xi k}\right|_{X_{k}}^{2}
\end{aligned}
$$

or

$$
\left|S\left(v_{\varepsilon \xi k}\right)-S\left(\widehat{v}_{\varepsilon \xi k}\right)\right|_{X_{k}}^{2} \leq\left[2 C_{3} C_{1 \varepsilon} k+8 C_{3} C_{2 \varepsilon} a^{4} k\right] T_{k}\left|v_{\varepsilon \xi k}-\widehat{v}_{\varepsilon \xi k}\right|_{X_{k}}^{2},
$$


Choosing $a^{2}>2 E_{0}$ where $E_{0}$ is defined by (3.22) and

$$
T_{k}=T_{k}(\varepsilon)=\min \left\{\frac{1}{2} ;\left(4 C_{0} C_{1 \varepsilon} k a^{2}\right)^{-1} ;\left(4 C_{3} C_{1 \varepsilon} k+16 C_{3} C_{2 \varepsilon} a^{4} k\right)^{-1}\right\}
$$

it follows, from (3.9), (3.16) and noting that $E_{0} \geq E_{0, \varepsilon}$, that $S_{k}$ has a unique fixed point $v_{\varepsilon \xi k}$ in $B_{a}$. Therefore the truncated problem has unique solution on $\left[0, T_{k}\right]$.

In order to pass the limit when $k \rightarrow \infty$ in the truncated problem we need to establish priori estimates:

\section{FIRST A PRIORI ESTIMATE}

Taking the scalar product in $\mathcal{H}_{0}$ of both sides of (3.2) with $2 v_{\varepsilon \xi k}^{\prime}(t)$, integrating from 0 to $t \leq T_{k}$ and using the hypothesis (2.2) we get:

$$
\left\{\begin{array}{l}
\left|\sqrt{K_{1 \xi}} v_{\varepsilon \xi k}^{\prime}(t)\right|_{0}^{2}+\int_{0}^{t}\left(2 K_{2}(s)+K_{1}^{\prime}(s)\right)\left|v_{\varepsilon \xi k}^{\prime}(s)\right|_{0}^{2} d s+\left|v_{\varepsilon \xi k}(t)\right|_{1 / 2}^{2}+ \\
+\widehat{M}\left(t,\left|v_{\varepsilon \xi k}(t)\right|_{\frac{1}{2}}^{2}\right) \leq\left|\sqrt{K_{1}(0)+\xi} v_{1 \varepsilon}\right|_{0}^{2}+\left|v_{0 \varepsilon}\right|_{\frac{1}{2}}^{2}+ \\
+\widehat{M}\left(0,\left|v_{0 \varepsilon}\right|_{\frac{1}{2}}^{2}\right)+\frac{1}{\rho} \int_{0}^{t}\left|g_{\varepsilon k}(s)\right|_{0}^{2} d s+\rho \int_{0}^{t}\left|v_{\varepsilon \xi k}^{\prime}(s)\right|_{0}^{2} d s
\end{array}\right.
$$

where $\rho>0$ is an arbitrary constant:

Using (2.6) and choosing $\rho=\delta$ we have

$$
\left\{\begin{aligned}
\left|\sqrt{K_{1 \varepsilon}} v_{\varepsilon \xi k}^{\prime}(t)\right|_{0}^{2} & +\int_{0}^{t}\left|v_{\varepsilon \xi k}^{\prime}(s)\right|_{0}^{2} d s+\left|v_{\varepsilon \xi k}(t)\right|_{0}^{2}+\widehat{M}\left(t,\left|v_{\varepsilon \xi k}(t)\right|_{\frac{1}{2}}^{2}\right) \leq \\
& \leq C_{4}\left[\left|v_{1 \varepsilon}\right|_{0}^{2}+\left|v_{0 \varepsilon}\right|_{\frac{1}{2}}^{2}+\widehat{M}\left(0,\left|v_{0 \varepsilon}\right|_{\frac{1}{2}}^{2}\right)+\left|g_{\varepsilon}\right|_{2,0}^{2}\right]
\end{aligned}\right.
$$

where $C_{4}>0$ independs of $0<\varepsilon \leq 1,0<\xi \leq 1, k \in \mathbb{N}, t \in\left[0, T_{k}\right]$ and

$$
\widehat{M}(t, \eta)=\int_{0}^{\eta} M(t, \tau) d \tau
$$

We have the following estimates 


$$
\begin{aligned}
& \left|v_{0 \varepsilon}\right|_{\frac{1}{2}}^{2}=\left|A_{\varepsilon}^{\frac{1}{2}} u_{0}\right|^{2} \leq\left|u_{0}\right|^{2}+\left|A^{\frac{1}{2}} u_{0}\right|^{2}, \quad 0<\varepsilon \leq 1 \\
& \left|v_{1 \varepsilon}\right|_{0}^{2}=\left|\mathcal{U}_{\varepsilon}\left(u_{1}\right)\right|_{2,0}^{2}=\left|u_{1}\right|^{2}, \quad 0<\varepsilon \leq 1 \\
& \left|g_{\varepsilon}\right|_{2,0}^{2}=\left|\mathcal{U}_{\varepsilon}(f)\right|_{2,0}^{2}=|f|_{L^{2}(0, T ; H)}^{2}, \quad 0<\varepsilon \leq 1 .
\end{aligned}
$$

Substituting (3.18)-(3.20) into (3.17) we get

$$
\left|\sqrt{K_{1 \xi}} v_{\varepsilon \xi k}^{\prime}(t)\right|_{0}^{2}+\int_{0}^{t}\left|v_{\varepsilon \xi k}^{\prime}(s)\right|_{0}^{2} d s+\left|v_{\varepsilon \xi k}(t)\right|_{\frac{1}{2}}^{2} \leq E_{0}
$$

where

$$
E_{0}=C_{4}\left[\left|u_{1}\right|^{2}+\left|u_{0}\right|^{2}+\left|A^{\frac{1}{2}} u_{0}\right|^{2}+|f|_{L^{2}(0, T ; H)}+\widehat{C}_{0}\right]
$$

and

$$
\widehat{C}_{0}=\max \left\{|\widehat{M}(0, \eta)| ; 0 \leq \eta \leq\left|u_{0}\right|^{2}+\left|A^{\frac{1}{3}} u_{0}\right|^{2}\right\}
$$

\section{SECOND A PRIORI ESTIMATE}

Taking the scalar product in $\mathcal{H}_{0}$ of both sides of (3.2) with $2 \lambda^{\frac{1}{2}} v_{\varepsilon \xi k}^{\prime}(t)$ and integrating from 0 to $t \leq T_{k}$, we obtain

$$
\begin{aligned}
& \left|\sqrt{K_{1 \varepsilon}} v_{\varepsilon \xi k}^{\prime}(t)\right|_{\frac{1}{4}}^{2}+\int_{0}^{t}\left(2 K_{2}+K_{1}^{\prime}\right)(s)\left|v_{\varepsilon \xi k}^{\prime}\right|_{\frac{1}{4}}^{2} d s+\left|v_{\varepsilon \xi k}(t)\right|_{\frac{1}{4}}^{2}+ \\
& \quad+M\left(t,\left|v_{\varepsilon \xi k}(t)\right|_{\frac{1}{2}}^{2}\right)\left|v_{\varepsilon \xi k}(t)\right|_{\frac{3}{4}}^{2}- \\
& \quad-\int_{0}^{t} \frac{\partial}{\partial s} M\left(s,\left|v_{\varepsilon \xi k}(s)\right|_{\frac{1}{2}}^{2}\right)\left|v_{\varepsilon \xi k}(s)\right|_{\frac{3}{4}}^{2} d s \leq
\end{aligned}
$$




$$
\begin{aligned}
& \leq\left|\sqrt{K_{1 \xi}(0)} v_{1 \varepsilon k}\right|_{\frac{1}{4}}^{2}+\left|v_{0 \varepsilon k}\right|_{\frac{3}{4}}^{2}+M\left(0,\left|v_{0 \varepsilon k}\right|_{\frac{1}{2}}^{2}\right)\left|v_{0 \varepsilon k}\right|_{\frac{3}{4}}^{2} \\
& +\int_{0}^{t}\left|M^{\prime}\left(s,\left|v_{\varepsilon \xi k}(s)\right|_{\frac{1}{2}}^{2}\right)\right|\left|\frac{d}{d s} v_{e \xi k}(s)\right|_{\frac{1}{2}}^{2}\left|v_{\varepsilon \xi k}(s)\right|_{\frac{3}{4}}^{2} d s+ \\
& +\int_{0}^{t} 2\left|g_{\varepsilon k}(s)\right|_{\frac{1}{4}}\left|v_{\varepsilon \xi k}^{t}(s)\right|_{\frac{1}{4}} d s .
\end{aligned}
$$

We have

$$
\left|\frac{d}{d s} v_{e \xi k}(s)\right|_{\frac{1}{2}}^{2} \leq 2\left|v_{e \xi k}^{\prime}(s)\right|_{\frac{1}{4}}\left|v_{\varepsilon \xi k}(s)\right|_{\frac{3}{4}}
$$

then, using the hypothesis (2.2) and (2.6) we get

$$
\begin{aligned}
& \left|\sqrt{K_{1 \xi}} v_{\varepsilon \xi k}^{\prime}(t)\right|_{\frac{1}{4}}^{2}+2 \delta \int_{0}^{t}\left|v_{\varepsilon \xi k}(s)\right|_{\frac{1}{4}}^{2} d s+\left|v_{\varepsilon \xi k}(t)\right|_{\frac{3}{4}}^{2}+ \\
& \quad+M\left(t,\left|v_{\varepsilon \xi k}(t)\right|_{\frac{1}{2}}^{2}\right)\left|v_{\varepsilon \xi k}(t)\right|_{\frac{3}{4}}^{2} \leq \\
& \quad \leq\left|\sqrt{K_{1 \xi}(0)} v_{1 \varepsilon}\right|_{\frac{3}{4}}^{2}+\left(C_{1}+1\right)\left|v_{0 \varepsilon}\right|_{\frac{3}{4}}^{2}+\frac{1}{\rho} \int_{0}^{t}\left|g_{\varepsilon}(s)\right|_{\frac{1}{4}}^{2} d s+ \\
& \quad+\frac{C_{2}}{\rho} \int_{0}^{t}\left|v_{\varepsilon \xi k}(s)\right|_{\frac{3}{4}}^{6} d s+\left(1+C_{2}\right) \rho \int_{0}^{t}\left|v_{\varepsilon \xi k}^{\prime}(s)\right|_{\frac{1}{4}}^{2} d s
\end{aligned}
$$

where $\rho>0$ is an arbitrary constant, and

$$
\begin{aligned}
& C_{1}=\max \left\{|M(0, \eta)| ; 0 \leq \eta \leq\left|u_{0}\right|^{2}+\left|A^{\frac{1}{2}} u_{0}\right|^{2}\right\} \\
& C_{2}=\max \left\{\left|M^{\prime}(t, \eta)\right| ; 0 \leq t \leq T ; 0 \leq \eta \leq\left|u_{0}\right|^{2}+\left|A^{\frac{1}{2}} u_{0}\right|^{2}\right\} .
\end{aligned}
$$

Choosing $\rho=\frac{\delta}{1+C_{2}}$ and from the estimates true for every $0<\varepsilon \leq 1$ 


$$
\begin{aligned}
& \left|v_{1 \varepsilon}\right|_{\frac{1}{4}}^{2}=\left|A_{\varepsilon}^{\frac{1}{4}} u_{1}\right|^{2} \leq\left|u_{1}\right|^{2}+\left|A^{\frac{1}{4}} u_{1}\right|^{2} \\
& \left|v_{0 \varepsilon}\right|_{\frac{3}{4}}^{2}=\left|A_{\varepsilon}^{\frac{3}{4}} u_{0}\right|^{2} \leq 2^{\frac{1}{2}}\left[\left|u_{0}\right|^{2}+\left|A^{\frac{3}{4}} u_{0}\right|^{2}\right] \\
& \left|g_{\varepsilon}\right|_{2, \frac{1}{4}}^{2}=\left|U_{\varepsilon}(f)\right|_{2, \frac{1}{4}}^{2} \leq|f|_{L^{2}(0, T ; H)}^{2}+\left|A^{\frac{1}{4}} f\right|_{L^{2}(0, T ; H)}^{2}
\end{aligned}
$$

we obtain:

$$
\begin{aligned}
& \left|\sqrt{K_{1 \xi}} v_{\varepsilon \xi k}^{\prime}(t)\right|_{\frac{1}{4}}^{2}+\int_{0}^{t}\left|v_{\varepsilon \xi k}^{\prime}(s)\right|_{\frac{1}{4}}^{2} d s+\left|v_{\varepsilon \xi k}(t)\right|_{\frac{3}{4}}^{2}+ \\
& +M\left(t,\left|v_{\varepsilon \xi k}(t)\right|_{\frac{1}{2}}^{2}\right)\left|v_{\varepsilon \xi k}(t)\right|_{\frac{3}{4}}^{2} \leq E_{1}+C_{5} \int_{0}^{t}\left|v_{\varepsilon \xi k}(s)\right|_{\frac{3}{4}}^{6} d s
\end{aligned}
$$

where

$$
\begin{aligned}
E_{1}= & C_{4}\left[\left(\left|u_{1}\right|^{2}+\left|A^{\frac{1}{2}} u_{1}\right|^{2}\right)+2^{\frac{1}{2}}\left(\left|u_{0}\right|^{2}+\left|A^{\frac{3}{4}} u_{0}\right|^{2}\right)\left(1+C_{1}\right)+\right. \\
& \left.+|f|_{L^{2}(0, T ; H)}^{2}+\left|A^{\frac{1}{4}} f\right|_{L^{2}(0, T ; H)}^{2}\right]
\end{aligned}
$$

and $C_{4}, C_{5}>0$ are constants independents of $0<\varepsilon \leq 1,0<\xi \leq 1$, $k \in \mathbf{N}$ and $t \in\left[0, T_{k}\right]$.

$$
\begin{aligned}
& \text { If } h_{\varepsilon \xi k}(t)=\left|v_{\varepsilon \xi k}(t)\right|_{\frac{3}{4}}^{2}, 0 \leq t \leq T_{k} \text {, from inequality (3.23) we obtain, } \\
& \quad 0 \leq h_{\varepsilon \xi k}(t) \leq E_{1}+C_{5} \int_{0}^{t} h_{\varepsilon \xi k}(s) d s, \quad 0 \leq t \leq T_{k}
\end{aligned}
$$

If $\theta_{\varepsilon \xi k}(t)=\int_{0}^{t} h_{\varepsilon \xi k}^{3}(s) d s$ then $\theta_{\varepsilon \xi k}^{\prime}(t) \leq\left(E_{1}+C_{5} \theta_{\varepsilon \xi k}(t)\right)^{3}$ and $E_{1}+$ $C_{5} \theta_{\varepsilon \xi k}(t) \leq\left\{\frac{E_{1}^{2}}{1-2 E_{1}^{2} C_{5} t}\right\}^{\frac{1}{2}}, 0 \leq t \leq T_{k}$. 

have

Taking $T^{*}=\frac{1}{2 E_{1}^{2} C_{5}}$ and $0<T_{0}<T^{*}, T_{0}$ a fixed real number, we

$$
\left|\sqrt{K_{1 \xi}} v_{\varepsilon \xi k}^{\prime}(t)\right|_{\frac{1}{4}}^{2}+\int_{0}^{t}\left|v_{\varepsilon \xi k}^{\prime}(s)\right|_{\frac{1}{4}}^{2} d s+\left|v_{\varepsilon \xi k}(t)\right|_{\frac{3}{4}}^{2} \leq E_{2}
$$

independent of $k \in \mathbb{N}, 0<\varepsilon \leq 1,0<\xi \leq 1$ and $t \in\left[0, T_{k}\right], 0<T_{k} \leq$ $T_{0}<T^{*}$, where $E_{2}=\left\{\frac{E_{1}^{2}}{1-2 E_{1}^{2} C_{5} T_{0}}\right\}^{\frac{1}{2}}$.

From the estimate (3.25) it follows that the local solution $v_{\varepsilon \xi k}$ can be extended to the hole interval $\left[0, T_{0}\right], T_{0}$ independent of $\varepsilon, \xi$ and $k$. In this interval the estimate (3.25) holds for all $k \in \mathbb{N}, 0<\varepsilon \leq 1,0<\xi \leq 1$ and $0<t \leq T_{0}$.

\section{LIMIT OF THE TRUNCATED SOLUTIONS}

The solution $v_{\varepsilon \xi k}$ of problem (3.2) satisfies

$$
\begin{gathered}
\left(K_{\left.1 \xi v_{\varepsilon \xi k}^{\prime}\right)^{\prime}+} K_{2} v_{\varepsilon \xi k}^{\prime}+\lambda v_{\varepsilon \xi k}+M\left(\cdot,\left|v_{\varepsilon \xi k}\right|_{\frac{1}{2}}^{2}\right) \lambda v_{\varepsilon \xi k}=g_{\varepsilon k}\right. \\
\text { in the sense of } L^{2}\left(0, T_{0} ; \mathcal{H}_{-\frac{1}{4}}\right) .
\end{gathered}
$$

From $v_{\varepsilon \xi k} \in L^{\infty}\left(0, T_{0} ; \mathcal{H}_{\frac{3}{4}}\right)$ and $v_{\varepsilon \xi k}^{\prime} \in L^{2}\left(0, T_{0} ; \mathcal{H}_{\frac{1}{4}}\right)$ it follows that $v_{\varepsilon \xi k} \in C^{0}\left(\left[0, T_{0}\right] ; \mathcal{H}_{\frac{2}{2}}\right)$ which implies that the function $\varphi_{\varepsilon \xi k}(t)=$ $\left|v_{\varepsilon \xi k}(t)\right|_{\frac{1}{2}}^{2}$ is continuous on $\left[0, T_{0}\right]$.

From (3.21) we have that

$$
\varphi_{\varepsilon \xi k}(t) \leq E_{0}, \quad \forall k \in \mathbb{N}, 0<\varepsilon \leq 1,0<\xi \leq 1,0 \leq t \leq \mathrm{T}_{0}
$$


Given $t_{0}, t_{1} \in\left[0, T_{0}\right]$ we obtain

$$
\begin{aligned}
\left|\varphi_{\varepsilon \xi k}\left(t_{1}\right)-\varphi_{\varepsilon \xi k}\left(t_{0}\right)\right| & \leq C_{6}\left|v_{\varepsilon \xi k}\left(t_{1}\right)-v_{\varepsilon \xi k}\left(t_{0}\right)\right|_{\frac{1}{4}} \leq \\
& \leq C_{6} \int_{t_{0}}^{t_{1}}\left|v_{\varepsilon \xi k}^{\prime}(s)\right|_{\frac{1}{4}} d s \leq C_{7}\left|t_{1}-t_{0}\right|^{\frac{1}{2}}
\end{aligned}
$$

for every $k \in \mathbf{N}, 0<\varepsilon \leq 1,0<\xi \leq 1, \mathbf{t}_{0}, \mathrm{t}_{1} \in\left[0, \mathrm{~T}_{0}\right]$.

As a consequence of (4.2) and (4.3) and the Arzéla-Áscoli theorem, exists a function $\varphi_{\varepsilon \xi} \in C^{0}\left(\left[0, T_{0}\right], \mathbb{R}\right)$ and a subsequence of $\left(\varphi_{\varepsilon \xi k}\right)_{k \in \mathbb{N}}$, still denoted by $\left(\varphi_{\varepsilon \xi k}\right)_{k \in \mathbb{N}}$, such that

$$
\varphi_{\varepsilon \xi k} \rightarrow \varphi_{\varepsilon \xi} \text { in } C^{0}\left(\left[0, T_{0}\right], \mathbb{R}\right) \text { when } \mathbf{k} \rightarrow \infty .
$$

By the assumption $M \in C^{1}([0, T] \times[0,+\infty[, \mathbb{R})$ it follows

$$
M\left(\cdot,\left|v_{\varepsilon \xi k}(\cdot)\right|_{\frac{1}{2}}^{2}\right) \rightarrow M\left(\cdot, \varphi_{\varepsilon \xi}(\cdot)\right) \text { in } C^{0}\left(\left[0, T_{0}\right], \mathbb{R}\right) .
$$

Therefore, from this convergence and estimate (3.25) we obtain

$$
\begin{aligned}
& v_{\varepsilon \xi k} \rightarrow v_{\varepsilon \xi} \text { in } L^{\infty}\left(0, T_{0} ; \mathcal{H}_{\frac{3}{4}}\right) \quad \text { weak star } \\
& v_{\varepsilon \xi k}^{\prime} \rightarrow v_{\varepsilon \xi}^{\prime} \text { in } L^{2}\left(0, T_{0} ; \mathcal{H}_{\frac{1}{4}}\right) \quad \text { weakly } \\
& \sqrt{K_{1 \xi}} v_{\varepsilon \xi k}^{\prime} \rightarrow \sqrt{K_{1 \xi}} v_{\varepsilon \xi}^{\prime} \text { in } L^{\infty}\left(0, T_{0} ; \mathcal{H}_{\frac{1}{t}}\right) \quad \text { weak star } \\
& M\left(\cdot,\left|v_{\varepsilon \xi k}(\cdot)\right|_{\frac{1}{2}}^{2}\right) \rightarrow M\left(\cdot, \varphi_{\varepsilon \xi}(\cdot)\right) \text { in } C^{0}\left(\left[0, T_{0}\right], \mathbb{R}\right)
\end{aligned}
$$

From the convergences (4.6)-(4.9) and observing that $g_{\varepsilon k} \rightarrow g_{\varepsilon}$ in $L^{2}\left(0, T_{0} ; \mathcal{H}_{\frac{1}{4}}\right)$, taking the limit in (4.1) when $k \rightarrow \infty$ we obtain: 


$$
\left\{\begin{array}{l}
\left(K_{1 \xi} v_{\varepsilon \xi}^{\prime}\right)^{\prime}+K_{2} v_{\varepsilon \xi}^{\prime}+\lambda v_{\varepsilon \xi}+M\left(\cdot, \varphi_{\varepsilon \xi}(\cdot)\right) \lambda v_{\varepsilon \xi}=g_{\varepsilon} \\
\text { in the sense of } L^{2}\left(0, T_{0} ; \mathcal{H}_{-\frac{1}{4}}\right) \\
v_{\varepsilon \xi}(0)=v_{0 \varepsilon}, \quad v_{\varepsilon \xi}^{\prime}(0)=v_{1 \varepsilon}^{\prime} .
\end{array}\right.
$$

Lemma 4.1. If $v_{\varepsilon \xi}$ is the field that satisfies (4.10) then $\varphi_{\varepsilon \xi}(t)=$ $\left|v_{\varepsilon \xi}(t)\right|_{\frac{1}{2}}^{2}$ comes up for every $t \in\left[0, T_{0}\right]$. lem

Proof. We consider $w_{\varepsilon \xi k}=v_{\varepsilon \xi k}-v_{\varepsilon \xi}$ then $w_{\varepsilon \xi k}$ satisfies the prob-

$$
\begin{aligned}
& \left\{\begin{array}{l}
\left(K_{1 \xi} w_{\varepsilon \xi k}^{\prime}\right)^{\prime}+K_{2} w_{\varepsilon \xi k}^{\prime}+\lambda w_{\varepsilon \xi k}+M\left(\cdot,\left|v_{\varepsilon \xi k}(\cdot)\right|_{\frac{1}{2}}^{2}\right) \lambda v_{\varepsilon \xi k}- \\
-M(\cdot, \varphi(\cdot)) \lambda v_{\varepsilon \xi}=g_{\varepsilon k}-g_{e} \text { in } L^{2}\left(0, T_{0} ; \mathcal{H}_{-\frac{1}{4}}\right)
\end{array}\right. \\
& \left\{\begin{array}{l}
w_{\varepsilon \xi k}(0)=v_{0 \varepsilon k}-v_{0 \varepsilon} \rightarrow 0 \text { in } \mathcal{H}_{\frac{3}{4}}, \text { strongly, when } k \rightarrow \infty \\
w_{\varepsilon \xi k}^{\prime}(0)=v_{1 \varepsilon k}-v_{1 \varepsilon} \rightarrow 0 \text { in } \mathcal{H}_{\frac{1}{4}}, \text { strongly, when } k \rightarrow \infty
\end{array}\right.
\end{aligned}
$$

Taking the duality $\langle,\rangle_{-\frac{1}{4}, \frac{1}{4}}$ of both sides of (4.11) with $2 w_{\varepsilon \xi k}^{\prime}$ we get

$$
\left\{\begin{array}{l}
\left\langle\left(K_{1 \xi} w_{\varepsilon \xi k}^{\prime}(t)\right)^{\prime}, 2 w_{\varepsilon \xi k}^{\prime}(t)\right\rangle+\left\langle K_{2} w_{\varepsilon \xi k}^{\prime}(t), 2 w_{\varepsilon \xi k}^{\prime}(t)\right\rangle+ \\
+\left\langle\lambda w_{\varepsilon \xi k}(t), 2 w_{\varepsilon \xi k}^{\prime}(t)\right\rangle+M\left(t,\left|v_{\varepsilon \xi k}(t)\right|_{\frac{1}{2}}^{2}\right)\left\langle w_{\varepsilon \xi k}(t), 2 w_{\varepsilon \xi k}^{\prime}(t)\right\rangle= \\
=\left\langle g_{\varepsilon k}(t)-g_{\varepsilon}(t), 2 w_{\varepsilon \xi k}^{\prime}(t)\right\rangle+[M(t, \varphi(t))- \\
\left.-M\left(t,\left|v_{\varepsilon \xi k}(t)\right|_{\frac{1}{2}}^{2}\right)\right]\left(\lambda v_{\varepsilon \xi}(t), 2 w_{\varepsilon \xi k}^{\prime}(t)\right\rangle .
\end{array}\right.
$$

We have 


$$
\left\{\begin{array}{l}
\frac{d}{d t}\left|\sqrt{K_{1 \xi}} w_{\varepsilon \xi k}^{\prime}(t)\right|_{0}^{2}=2\left\langle\sqrt{K_{1 \xi}} w_{\varepsilon \xi k}^{\prime}(t), \sqrt{K_{1 \xi}} w_{\varepsilon \xi k}^{\prime}(t)\right\rangle= \\
=\left\langle K_{1 \xi} w_{\varepsilon \xi k}^{\prime \prime}(t), 2 w_{\varepsilon \xi k}^{\prime}(t)\right\rangle_{-\frac{1}{\xi}, \frac{1}{4}}+\left\langle K_{1}^{\prime} w_{\varepsilon \xi k}^{\prime}(t), w_{\varepsilon \xi k}^{\prime}(t)\right\rangle_{-\frac{1}{4}, \frac{1}{4}}
\end{array}\right.
$$

and

$$
\left\{\begin{array}{l}
\frac{d}{d t}\left[M\left(t,\left|v_{\varepsilon \xi k}(t)\right|_{\frac{1}{2}}^{2}\right)\left|v_{\varepsilon \xi k}(t)\right|_{\frac{1}{2}}^{2}\right]=M\left(t,\left|v_{\varepsilon \xi k}(t)\right|_{\frac{1}{2}}^{2}\right) \frac{d}{d t}\left[\left.w_{\varepsilon \xi k}(t)\right|_{\frac{1}{2}} ^{2}+\right. \\
+\left|w_{\varepsilon \xi k}(t)\right|_{\frac{1}{2}}^{2}\left[\frac{\partial}{\partial t} M\left(t,\left|v_{\varepsilon \xi k}(t)\right|_{\frac{1}{2}}^{2}\right)+M^{\prime}\left(t,\left.v_{\varepsilon \xi k}(t)\right|_{\frac{1}{2}} ^{2}\right) \frac{d}{d t}\left|v_{\varepsilon \xi k}(t)\right|_{\frac{1}{2}}^{2}\right]
\end{array}\right.
$$

where $M^{\prime}(t, \eta)=\frac{\partial}{\partial \eta} M(t, \eta)$.

Substituting (4.14), (4.15) into (4.13) and integrating from 0 to $t \leq T_{0}$ we obtain

$$
\begin{aligned}
& \left|\sqrt{K_{1 \xi}} w_{\varepsilon \xi k}^{\prime}(t)\right|_{0}^{2}+\int_{0}^{t}\left(2 K_{2}+K_{1}^{\prime}\right)(s)\left|w_{\varepsilon \xi k}^{\prime}(s)\right|_{0}^{2} d s+\left|w_{\varepsilon \xi k}(t)\right|_{\frac{1}{2}}^{2}+ \\
& +M\left(t,\left|v_{\varepsilon \xi k}(t)\right|_{\frac{1}{2}}^{2}\right)\left|w_{\varepsilon \xi k}(t)\right|_{\frac{1}{2}}^{2}-\int_{0}^{t} \frac{\partial}{\partial s} M\left(s,\left.v_{\varepsilon \xi k}(s)\right|_{\frac{1}{2}} ^{2}\right)\left|w_{\varepsilon \xi k}(s)\right|_{\frac{1}{2}}^{2} d s \leq \\
& \leq E_{3 \varepsilon k}+\left.\left.\int_{0}^{t}\left|M^{\prime}\left(s,\left|v_{\varepsilon \xi k}(s)\right|_{\frac{1}{2}}^{2}\right)\right|\left|\frac{d}{d s}\right| v_{\varepsilon \xi k}(s)\right|_{\frac{1}{2}} ^{2}|| w_{\varepsilon \xi k}(s)\right|_{\frac{1}{2}} ^{2} d s+ \\
& +2 \int_{0}^{t}\left|M(s, \varphi(s))-M\left(s,\left|v_{\varepsilon \xi k}(s)\right|_{\frac{1}{2}}^{2}\right)\right|\left|v_{\varepsilon \xi \xi}(s)\right|_{\frac{3}{4}}\left|w_{\varepsilon \xi k}^{\prime}(s)\right|_{\frac{1}{4}} d s+ \\
& +2 \int_{0}^{t}\left|g_{\varepsilon k}(s)-g_{\varepsilon}(s)\right|\left|w_{\varepsilon \xi k}^{\prime}(s)\right| d s .
\end{aligned}
$$

where 
$E_{3 \varepsilon k}=\left|\sqrt{K_{1 \xi}(0)}\left(v_{1 \varepsilon k}-v_{1 \varepsilon}\right)\right|_{0}^{2}+\left|v_{0 \varepsilon k}-v_{1 \varepsilon}\right|_{\frac{1}{2}}^{2}+M\left(0,\left|v_{0 \varepsilon k}\right|_{\frac{1}{2}}^{2}\right)\left|v_{0 \varepsilon k}-v_{0 \varepsilon}\right|_{0}^{2}$

Considering the hypothesis (2.2) and estimates (3.25) we have

$$
\left\{\begin{array}{l}
\left|\sqrt{K_{1 \xi}} w_{\varepsilon \xi k}^{\prime}(t)\right|_{0}^{2}+\int_{0}^{t}\left|w_{\varepsilon \xi k}^{\prime}(s)\right|_{0}^{2} d s+\left|w_{\varepsilon \xi k}(t)\right|_{\frac{1}{2}}^{2}+ \\
+M\left(t,\left|v_{\varepsilon \xi k}(t)\right|_{\frac{1}{2}}^{2}\right)\left|w_{\varepsilon \xi k}(t)\right|_{\frac{1}{2}}^{2} \leq \widehat{E}_{3 \varepsilon k}+ \\
+C_{8} \int_{0}^{t}\left[1+\left|v_{\varepsilon \xi k}^{\prime}(s)\right|_{\frac{1}{4}}^{2} d s\right]\left|w_{\varepsilon \xi k}(s)\right|_{\frac{1}{2}}^{2} d s
\end{array}\right.
$$

where $\widehat{E}_{3 \varepsilon k} \rightarrow 0$ when $k \rightarrow \infty$ independently of $t \in\left[0, T_{0}\right]$.

The Gronwall's inequality and (3.17) imply that

$$
\lim _{k \rightarrow \infty}\left|w_{\varepsilon \xi k}(t)\right|_{\frac{1}{2}}=0 \text { uniformly in }\left[0, T_{0}\right]
$$

Finally, given $t \in\left[0, T_{0}\right]$ we have

$$
\left\{\begin{array}{l}
\left.\left|\varphi_{\varepsilon \xi}(t)-\right| v_{\varepsilon \xi}(t)\right|_{\frac{1}{2}} ^{2}|\leq| \varphi_{\varepsilon \xi}(t)-\varphi_{\varepsilon \xi k}(t)|+| \varphi_{\varepsilon \xi k}(t)-\left|v_{\varepsilon \xi}(t)\right|_{\frac{1}{2}}^{2} \mid \leq \\
\leq\left|\varphi_{\varepsilon \xi}(t)-\varphi_{\varepsilon \xi k}(t)\right|+\left[\left|v_{\varepsilon \xi k}(t)\right|_{\frac{1}{2}}+\left|v_{\varepsilon \xi}(t)\right|_{\frac{1}{2}}\right]\left|w_{\varepsilon \xi k}(t)\right|_{\frac{1}{2}}^{2} .
\end{array}\right.
$$

Taking the limit when $k \rightarrow \infty$ in (4.19) and using (4.4) and (4.18), we have from (4.19)

$$
\varphi_{\varepsilon \xi}(t)=\left|v_{\varepsilon \xi}(t)\right|_{\frac{1}{2}}^{2} ; \quad \forall t \in\left[0, T_{0}\right] .
$$

From the estimates (3.25) and the Uniform Boundedness Theorem we obtain 


$$
\left\{\begin{array}{l}
\left|v_{\varepsilon \xi}(t)\right|_{\frac{3}{4}}^{2} \leq C_{9} \\
\int_{0}^{t}\left|v_{\varepsilon \xi}^{\prime}(s)\right|_{\frac{1}{4}}^{2} d s \leq C_{9} \\
\left.\sqrt{K_{1 \xi}} v_{\varepsilon \xi}^{\prime}(t)\right|_{\frac{1}{4}} ^{2} \leq C_{9}
\end{array}\right.
$$

where $C_{9}>0$ is a constant independent of $0<\varepsilon \leq 1,0<\xi \leq 1$ and $t \in\left[0, T_{0}\right]$.

From the above estimates we conclude that exists a vector field $v_{\varepsilon}$ such that

$$
\left\{\begin{array}{l}
v_{\varepsilon \xi} \rightarrow v_{\varepsilon} \text { in } L^{\infty}\left(0, T_{0} ; \mathcal{H}_{\frac{3}{4}}\right) \text { weak star } \\
v_{\varepsilon \xi}^{\prime} \rightarrow v_{\varepsilon}^{\prime} \text { in } L^{2}\left(0, T_{0} ; \mathcal{H}_{\frac{1}{4}}\right) \text { weakly } \\
\sqrt{K_{1 \xi}} v_{\varepsilon \xi}^{\prime} \rightarrow \sqrt{K_{1}} v_{\varepsilon}^{\prime} \text { in } L^{2}\left(0, T_{0} ; \mathcal{H}_{\frac{2}{4}}\right) \text { weakly }
\end{array}\right.
$$

Using the same arguments used to proof (4.5) we conclude that

$$
M\left(\cdot,\left|v_{\varepsilon \xi}(\cdot)\right|_{\frac{1}{2}}^{2}\right) \rightarrow M\left(\cdot,\left|v_{\varepsilon}(\cdot)\right|_{\frac{1}{2}}^{2}\right) \text { in } C^{0}\left(\left[0, T_{0}\right], \mathbb{R}\right) .
$$

Taking the limit in (4.10), when $\xi \rightarrow 0^{+}$we obtain

$$
\left\{\begin{array}{l}
\left(K_{1} v_{\varepsilon}^{\prime}\right)^{\prime}+K_{2} v_{\varepsilon}^{\prime}+\lambda v_{\varepsilon}+M\left(,\left|v_{\varepsilon}\right|_{\frac{1}{2}}^{2}\right) \lambda v_{\varepsilon}=g_{\varepsilon} \text { in } L^{2}\left(0, T_{0} ; \mathcal{H}_{-\frac{1}{4}}\right) \\
v_{\varepsilon}(0)=v_{0 \varepsilon}, \quad v_{\varepsilon}^{\prime}(0)=v_{1 \varepsilon} .
\end{array}\right.
$$

The vector function $u_{\varepsilon}:\left[0, T_{0}\right] \rightarrow H$ defined by $u_{\varepsilon}(t)=\mathcal{U}_{\varepsilon}^{-1}\left(v_{\varepsilon}(t)\right)$ satisfies (2.10) and from Uniform Boundedness Theorem we obtain the estimates 


$$
\left\{\begin{array}{l}
\left|u_{\varepsilon}(t)\right|^{2}+\left|A_{\varepsilon}^{\frac{3}{4}} u_{\varepsilon}(t)\right|^{2} \leq C_{10} \\
\int_{0}^{T_{0}}\left|u_{\varepsilon}^{\prime}(t)\right|^{2} d t+\int_{0}^{T_{0}}\left|A_{\varepsilon}^{\frac{1}{t}} u_{\varepsilon}^{\prime}(t)\right|^{2} d t \leq C_{10} \\
\left|\sqrt{K_{1}} u_{\varepsilon}^{\prime}(t)\right|^{2}+\left|A_{\varepsilon}^{\frac{1}{4}}\left(\sqrt{K_{1}} u_{\varepsilon}^{\prime}\right)(t)\right|^{2} \leq C_{10}
\end{array}\right.
$$

where $C_{10}>0$ is a constant independent of $\varepsilon$ and $t \in\left[0, T_{0}\right]$.

From the estimates (4.24) we conclude that the family of continuous functions $\varphi_{\varepsilon}(t)=\left|A_{\varepsilon}^{\frac{1}{2}} u_{\varepsilon}(t)\right|^{2}$ satisfies the hypothesis of the Arzelá-Áscoli theorem, therefore, there exists a continuous function $\varphi \in C^{0}\left(\left[0, T_{0}\right], \mathbb{R}\right)$ and a subnet of $\left(u_{\varepsilon}\right)_{0<\varepsilon \leq 1}$, which we still represent by $\left(u_{\varepsilon}\right)_{0<\varepsilon \leq 1}$, such that

$$
\left\{\begin{array}{l}
u_{\varepsilon} \rightarrow u \text { in } L^{\infty}\left(0, T_{0} ; D\left(A^{\frac{3}{4}}\right)\right) \text { weak star } \\
u_{\varepsilon}^{\prime} \rightarrow u^{\prime} \text { in } L^{2}\left(0, T_{0} ; D\left(A^{\frac{1}{4}}\right)\right) \text { weakly } \\
\sqrt{K_{1}} u_{\varepsilon}^{\prime} \rightarrow \sqrt{K_{1}} u^{\prime} \text { in } L^{2}\left(0, T_{0} ; D\left(A^{\frac{1}{4}}\right)\right) \text { weakly } \\
M\left(t,\left|A_{\varepsilon}^{\frac{1}{2}} u_{\varepsilon}(t)\right|^{2}\right) \rightarrow M(t, \varphi(t)) \text { in } C^{0}\left(\left[0, T_{0}\right] ; \mathbb{R}\right)
\end{array}\right.
$$

Using the convergences (4.25) we can take the limit in (2.10), when $\varepsilon \rightarrow 0^{+}$, and we obtain

$$
\left\{\begin{array}{l}
\left(K_{1} u^{\prime}\right)^{\prime}+K_{2} u^{\prime}+A u+M(\cdot ; \varphi(\cdot))=f \text { in } L^{2}\left(0, T_{0} ; D\left(A^{\frac{1}{1}}\right)^{\prime}\right) \\
u(0)=u_{0}, \quad u^{\prime}(0)=u_{1} .
\end{array}\right.
$$

To complete the proof of Theorem 2.1 it is sufficient to prove that $\varphi(t)=\left|A^{\frac{1}{2}} u(t)\right|^{2}, \forall t \in\left[0, T_{0}\right]$.

Lemma 4.2. If $u$ is the field that satisfies (4.26) then $\varphi(t)=$ $\left|A^{\frac{1}{2}} u(t)\right|^{2}, \forall t \in\left[0, T_{0}\right]$. 
Proof. Let $z \in D\left(A^{\frac{1}{4}}\right)$ and $w_{c}=u_{\varepsilon}-u$. By (4.26) we obtain

$$
\left\{\begin{array}{l}
\frac{d}{d t}\left(K_{1} w_{\varepsilon}^{\prime}(t), z\right)+\left(K_{2} w_{\varepsilon}^{\prime}(t), z\right)+\left(A^{\frac{3}{4}} w_{\varepsilon}(t), A^{\frac{1}{4}} z\right)+ \\
+M\left(t, \varphi_{\varepsilon}(t)\right)\left(A^{\frac{3}{2}} u_{\varepsilon}(t), A^{\frac{1}{4}} z\right)-M(t, \varphi(t))\left(A^{\frac{3}{4}} u(t), A^{\frac{1}{4}} z\right)=0
\end{array}\right.
$$

for every $z \in D\left(A^{\frac{1}{4}}\right)$.

If we take $z=2 w_{\varepsilon}^{t}(t)$ we have

$$
\begin{gathered}
2\left(A^{\frac{3}{4}} w_{\varepsilon}, A^{\frac{1}{4}} w_{\varepsilon}^{\prime}\right)=2\left(A_{\varepsilon}^{\frac{3}{4}} w_{\varepsilon}, A_{\varepsilon}^{\frac{1}{4}} w_{\varepsilon}^{\prime}\right)-2 \varepsilon\left(w_{\varepsilon}, w_{\varepsilon}^{\prime}\right) \\
\frac{d}{d t}\left|A_{\varepsilon}^{\frac{1}{2}} w_{\varepsilon}(t)\right|^{2}=2\left(A_{\varepsilon}^{\frac{3}{4}} w_{\varepsilon}(t), A_{\varepsilon}^{\frac{1}{4}} w_{\varepsilon}^{\prime}(t)\right)
\end{gathered}
$$

and

$$
\left\{\begin{array}{l}
\frac{d}{d t}\left[M\left(t, \varphi_{\varepsilon}(t)\right)\left|A_{\varepsilon}^{\frac{1}{2}} w_{\varepsilon}(t)\right|^{2}\right]=M\left(t, \varphi_{\varepsilon}(t)\right) \frac{d}{d t}\left|A_{\varepsilon}^{\frac{1}{2}} w_{\varepsilon}(t)\right|^{2}+ \\
+\left|A_{\varepsilon}^{\frac{1}{2}} w_{\varepsilon}(t)\right|^{2}\left[2 M^{\prime}\left(t, \varphi_{\varepsilon}(t)\right)\left(A_{\varepsilon}^{\frac{3}{4}} u_{\varepsilon}(t), A_{\varepsilon}^{\frac{1}{4}} u_{\varepsilon}^{\prime}(t)\right)+\frac{\partial}{\partial t} M\left(t, \varphi_{\varepsilon}(t)\right)\right]
\end{array}\right.
$$

Substituting (4.28)-(4.30) into (4.27) we have

$$
\begin{aligned}
& \frac{d}{d t}\left[\left|\sqrt{K_{1}} w_{\varepsilon}^{\prime}(t)\right|^{2}+\left|A_{\varepsilon}^{\frac{1}{2}} w_{\varepsilon}(t)\right|^{2}+M\left(t, \varphi_{\varepsilon}(t)\right)\left|A_{\varepsilon}^{\frac{1}{2}} w_{\varepsilon}(t)\right|^{2}\right]+ \\
& \quad+\left(2 K_{2}+K_{1}^{\prime}\right)(t)\left|w_{\varepsilon}^{\prime}(t)\right|^{2}-\frac{\partial}{\partial t} M\left(t, \varphi_{\varepsilon}(t)\right)\left|A_{\varepsilon}^{\frac{1}{2}} w_{\varepsilon}(t)\right|^{2}= \\
& \quad=2 M^{\prime}\left(t, \varphi_{\varepsilon}(t)\right)\left(A_{\varepsilon}^{\frac{3}{4}} u_{\varepsilon}(t), A_{\varepsilon}^{\frac{1}{4}} u_{\varepsilon}^{\prime}(t)\right)\left|A_{\varepsilon}^{\frac{1}{2}} w_{\varepsilon}(t)\right|^{2}+ \\
& \quad+2\left[M(t, \varphi(t))-M\left(t, \varphi_{\varepsilon}(t)\right)\right]\left(A^{\frac{3}{4}} u(t), A^{\frac{1}{2}} w_{\varepsilon}^{\prime}(t)\right)+4 \varepsilon\left(w_{\varepsilon}(t), w_{\varepsilon}^{\prime}(t)\right)
\end{aligned}
$$


Integrating from 0 to $t \leq T_{0}$ and considering the hypothesis (2.2) and (2.6) we obtain

$$
\begin{aligned}
& \left|\sqrt{K_{1}} w_{\varepsilon}^{\prime}(t)\right|^{2}+ \\
& +2 \delta \int_{0}^{t}\left|w_{\varepsilon}^{\prime}(s)\right|^{2} d s+\left|A_{\varepsilon}^{\frac{1}{2}} w_{\varepsilon}(t)\right|^{2}+M\left(t, \varphi_{\varepsilon}(t)\right)\left|A_{\varepsilon}^{\frac{1}{2}} w_{\varepsilon}(t)\right|^{2} \leq \\
& \leq 2 \int_{0}^{t}\left|M^{\prime}(s, \varphi(s))\right|\left|A_{\varepsilon}^{\frac{3}{4}} u_{\varepsilon}(s)\right|\left|A_{\varepsilon}^{\frac{1}{t}} u_{\varepsilon}^{\prime}(s)\right|\left|A_{\varepsilon}^{\frac{1}{2}} w_{\varepsilon}^{\prime}(s)\right|^{2} d s+ \\
& +2 \int_{0}^{t}\left|M\left(s, \varphi_{\varepsilon}(s)\right)-M(s, \varphi(s))\right|\left|A^{\frac{3}{4}} u(s)\right|\left|A^{\frac{1}{4}} w_{\varepsilon}^{\prime}(s)\right| d s+ \\
& +4 \varepsilon \int_{0}^{t}\left|w_{\varepsilon}(s) \| w_{\varepsilon}^{\prime}(s)\right| d s .
\end{aligned}
$$

We observe that $\left|M^{\prime}(t, \varphi(t))\right|,\left|A_{\varepsilon}^{\frac{3}{4}} u_{\varepsilon}(t)\right|,\left|A^{\frac{3}{4}} u(t)\right|$ and $\left|A^{\frac{1}{4}} w_{\varepsilon}^{\prime}(t)\right|$ are bounded independent of $\varepsilon$ and $t \in\left[0, T_{0}\right]$. We also have that the terms $\left|M\left(t, \varphi_{\varepsilon}(t)\right)-M(t, \varphi(t))\right|$ and $4 \varepsilon \int_{0}^{t}\left|w_{\varepsilon}(s) \|\right| w_{\varepsilon}^{\prime}(s) \mid d s$ converge uniformly to zero in $\left[0, T_{0}\right]$ when $\varepsilon \rightarrow 0^{+}$.

From (4.31) we have

$$
\left|A_{\varepsilon}^{\frac{1}{2}} w_{\varepsilon}(t)\right|^{2} \leq \mathcal{O}(\varepsilon)+C_{11} \int_{0}^{t}\left[1+\left|A_{\varepsilon}^{\frac{1}{4}} u_{\varepsilon}^{\prime}(s)\right|^{2}\right]\left|A_{\varepsilon}^{\frac{1}{2}} w_{\varepsilon}(s)\right|^{2} d s
$$

where $C_{11}>0$ is a constant independent of $\varepsilon$ and $t$, and $\lim _{\varepsilon \rightarrow 0^{+}} \mathcal{O}(\varepsilon)=$ 0 , independent of $t \in\left[0, T_{0}\right]$.

From (4.32) and Gronwall lemma we have 


$$
\left|A_{\varepsilon}^{\frac{1}{2}} w_{\varepsilon}(t)\right|^{2} \rightarrow 0 \text { uniformly in }\left[0, T_{0}\right] \text {, when } \varepsilon \rightarrow 0^{+}
$$

Given $t \in\left[0, T_{0}\right]$ we have

$$
\left.|\varphi(t)-| A^{\frac{1}{2}} u(t)\right|^{2}|\leq| \varphi(t)-\varphi_{\varepsilon}(t)|+| \varphi_{\varepsilon}(t)-\left|A^{\frac{1}{2}} u(t)\right|^{2} \mid
$$

and

$$
\left\{\begin{array}{l}
\left.\left|\varphi_{\varepsilon}(t)-\right| A^{\frac{1}{2}} u(t)\right|^{2}|\leq|\left|A_{\varepsilon}^{\frac{1}{2}} u_{\varepsilon}(t)\right|^{2}-\left|A_{\varepsilon}^{\frac{1}{2}} u(t)\right|^{2} \mid+ \\
+\left.|| A_{\varepsilon}^{\frac{1}{2}} u(t)\right|^{2}-\left|A^{\frac{1}{2}} u(t)\right|^{2} \leq \\
\leq\left[\left|A_{\varepsilon}^{\frac{1}{2}} u_{\varepsilon}(t)\right|+\left|A_{\varepsilon}^{\frac{1}{2}} u(t)\right|\right]\left|A_{\varepsilon}^{\frac{1}{2}} w_{\varepsilon}(t)\right|+\varepsilon|u(t)|^{2}
\end{array}\right.
$$

Substituting (4.35) into (4.34) and observing that $\left|A_{\varepsilon}^{\frac{1}{2}} u_{\varepsilon}(t)\right|+$ $+\left|A_{\varepsilon}^{\frac{1}{2}} u(t)\right|$ is bounded independent of $\varepsilon$ and $t \in\left[0, T_{0}\right]$ we obtain

$$
\left.|\varphi(t)-| A^{\frac{1}{2}} u(t)\right|^{2}|\leq| \varphi(t)-\left.\varphi_{\varepsilon}(t)|+\varepsilon| u(t)\right|^{2}+C_{12}\left|A_{\varepsilon}^{\frac{1}{2}} w_{\varepsilon}(t)\right| .
$$

Since $\varphi_{\varepsilon} \rightarrow \varphi$ in $C^{0}\left(\left[0, T_{0}\right], \mathbb{R}\right)$ we obtain from (4.36) and (4.33) that $\varphi(t)=\left|A^{\frac{1}{2}} u(t)\right|^{2}$ when $\varepsilon \rightarrow 0^{+}$.

\section{APPLICATIONS}

(i) Let $\Omega$ be a bounded open set of $\mathbb{R}^{\mathbf{n}}$ with smooth boundary $\Gamma$ and $A=-\Delta$ the operator defined by the triple $\left\{H_{0}^{1}(\Omega) ; L^{2}(\Omega) ;(())_{H_{0}^{1}(\Omega)}\right\}$. As a consequence of Theorem 2.1 , we find a function $u:\left[0, T_{0}\right] \rightarrow L^{2}(\Omega)$ such that 


$$
\left\{\begin{array}{l}
\left(K_{1} u^{\prime}\right)^{\prime}+K_{2} u^{\prime}-\left[1+M\left(t, \int_{\Omega}|\nabla u(t, x)|^{2} d x\right)\right] \Delta u=f \\
u(0, x)=u_{0}(x), \quad \frac{\partial u}{\partial t}(0, x)=u_{1}(x), \quad x \in \Omega \\
\left.u=0 \text { on } \Sigma_{0}=\Gamma \times\right] 0, T_{0}[.
\end{array}\right.
$$

We observe that the example 5(i) can also be solved making use of compactness arguments which can not be applied to case 5(iii).

The importance of the diagonalization method is that it allows us to solve problems when $\Omega$ is a bounded open set of $\mathbb{R}^{n}$ and we have compactness condition, or in the case where $\Omega$ is unbounded and so there is no compactness condition.

(ii) Let $\Omega$ be as (i) and $B$ the operator defined by the triple $\left\{H^{1}(\Omega)\right.$; $\left.L^{2}(\Omega) ; a(u, v)\right\}$ where

$$
a(u, v)=\sum_{i, j=1}^{n} \int_{\Omega} a_{i j}(x) \frac{\partial u}{\partial x_{i}} \frac{\partial v}{\partial x_{j}} d x+\int_{\Omega} a_{0}(x) u v d x, u, v \in H^{1}(\Omega)
$$

with $a_{0}, a_{i j} \in L^{\infty}(\Omega), a_{i j}=a_{j i}, i, j=1, \cdots, n, a_{0}(x) \geq \alpha_{0}>0$ for almost $x \in \Omega$, and exists $\alpha>0$ such that $\Sigma_{i, j=1}^{n} a_{i j}(x) \xi_{i} \xi_{j} \geq \alpha|\xi|^{2}$, for almost $x \in \Omega$ and every $\xi \in \mathbb{R}^{\mathbf{n}}$.

The operator $B$ is $B=-\sum_{i, j=1}^{n} \frac{\partial}{\partial x_{i}}\left(a_{i j} \frac{\partial}{\partial x_{j}}\right)+a_{0} I$ with domain

$$
D(B)=\left\{u \in H^{2}(\Omega), \frac{\partial u}{\partial \nu_{B}}=0 \text { on } \Gamma\right\}
$$

where

$$
\frac{\partial u}{\partial \nu_{B}}=\sum_{i, j=1}^{n} a_{i j}(x) \frac{\partial u}{\partial x_{j}} \cos \left(\nu, x_{i}\right)
$$

$\nu$ is the outward normal to $\Gamma$.

Let $A$ be the operator $A=-\sum_{i, j=1}^{n} \frac{\partial}{\partial x_{i}}\left(a_{i j} \frac{\partial}{\partial x_{j}}\right)$ with domain $D(A)$ 
$=D(B) . \quad A$ is a self-adjoint operator and by Gauss lemma it follows that

$$
\begin{aligned}
(A u, u) & =\left(-\sum_{i, j=1}^{n} \frac{\partial}{\partial x_{i}}\left(a_{i j}(x) \frac{\partial u}{\partial x_{j}}\right), u\right)= \\
& =\int_{\Omega} \sum_{i, j=1}^{n} a_{i j}(x) \frac{\partial u}{\partial x_{j}} \frac{\partial u}{\partial x_{i}} d x- \\
& -\int_{\Gamma} \sum_{i, j=1}^{n} a_{i j}(x) \frac{\partial u}{\partial x_{j}} u \cos \left(\nu, x_{i}\right) d \Gamma \geq \\
& \geq \alpha|\nabla u|^{2}, \quad \forall u \in D(A) .
\end{aligned}
$$

If $\left(a_{i j}\right)_{n \times n}=I$ where $I$ is the identity matrix than $A=-\Delta$ and $D(A)=$ $\left\{u \in H^{2}(\Omega), \frac{\partial u}{\partial \nu}=0\right.$ on $\left.\Gamma\right\}$.

By Theorem 2.1 exists a function $u:\left[0, T_{0}\right] \rightarrow L^{2}(\Omega)$ such that

$$
\left\{\begin{array}{l}
\left(K_{1} u^{\prime}\right)^{\prime}+K_{2} u^{\prime}-\left[1+M\left(t, \int_{\Omega}|\nabla u(t, x)|^{2} d x\right)\right] \Delta u=f \\
u(0, x)=u_{0}(x), \quad \frac{\partial u}{\partial t}(0, x)=u_{1}(x) \quad x \in \Omega \\
\left.\frac{\partial u}{\partial \nu}=0 \text { on } \Sigma_{0}=\Gamma \times\right] 0, T_{0}[
\end{array}\right.
$$

iii) Let $\Omega=\mathbb{R}^{\mathrm{n}}$ and $A=-\Delta$ the operator of $L^{2}\left(\mathbb{R}^{\mathrm{n}}\right)$ with domain $D(A)=H^{2}\left(\mathbb{R}^{\mathrm{n}}\right)$.

By Theorem 2.1 exists a function $u:\left[0, T_{0}\right] \rightarrow L^{2}\left(\mathbb{R}^{\mathrm{n}}\right)$ such that

$$
\left\{\begin{array}{l}
\left(K_{1} u^{\prime}\right)^{\prime}+K_{2} u^{\prime}-\left[1+M\left(t, \int_{\mathbf{R}^{\mathrm{n}}}|\nabla u(t, x)|^{2} d x\right)\right] \Delta u=f \\
u(0, x)=u_{0}(x), \quad \frac{\partial u}{\partial t}(0, x)=u_{1}(x), \quad x \in \mathbb{R}^{\mathrm{n}}
\end{array}\right.
$$

We observe that when the abstract results are realized in the concret case (iii) with $K_{1}=1, K_{2}=0$ and $M$ depends only on $\int_{\mathbb{R}^{\mathbf{n}}}|\nabla u(t, x)|^{2} d x$, 
we obtain the results proved by the method of Fourier transform, by Dickey [6] and Menzala [19].

\section{Acknowledgements}

I would like to express my thanks to Professor L.A. Medeiros for his encouragement and valuable suggestions and to Professor E. Zuazua and the referee for the constructive remarks.

\section{References}

[1] Arosio, A. and Garavaldi, S., On the degenerate Kirchhoff string, Math. Appl. Science, Vol. 14 (1991), 177-195.

[2] Arosio, A. and Spagnolo, S., Global solution to the Cauchy problem for nonlinear hyperbolic equation, Nonlinear Partial Differential Equation and their Application, Collège de France seminar, Vol. 6, Edited by H. Brezis and J.L. Lions, Pitman, London (1984).

[3] Bensoussan, A., Lions, J.L. and Papanicolau, G., Perturbation et Augmentation des Conditions Initiales. Singular Perturbation and Boundary Layer Theory, Springer Verlag, Lyon, (1976).

[4] Bubnov, B.A., Goursat and Darboux problems for a class of hyperbolic equations, Siberian Math. Journal, Vol. 19, $\mathrm{N}^{\circ} 2$ (1978), 325-328.

[5] Bryukhanov, V.A., A mixed problem for a hyperbolic equation degenerate on a part of the boundary of a region, Differential Equations, Vol. 8, $\mathrm{N}^{\circ} 1$ (1972), 1-3.

[6] Carrier, G.F., On the vibrations problem of elastic string, Q.J. Appl. Math, Vol. 3 (1945), 151-165.

[7] Crippa, H.R., On local solutions of some mildly degenerate hyperbolic equations, Nonlinear Analysis, Theory, Methods and Applications, (to appear).

[8] Dickey, R.W., The initial value problem for a nonlinear semi-infinite string, Proc. Royal Soc. Edinburgh, 82 (1978), 19-26.

[9] Dixmier, J., Les Algèbres d'Opérateurs dans l'Espace Hilbertien, Gauthier Villars, Paris, (1957). 
[10] Ebihara, Y., Medeiros, L.A. and Milla Miranda, M., Local solution for a nonlinear degenerate hyperbolic equation, Nonlinear Analysis, 10 (1986), 27-40.

[11] Ferreira, J., On weak solutions of semi-linear hyperbolic-parabolic equation, Matematickỹ Ústav Ćsskokvenské Academiẽ Praze, (to appear).

[12] Gadzhiev, M.M., A mixed problem for a nonlinear hyperbolicparabolic equation, Differential Equations, Vol. 13, $\mathrm{N}^{0} 2$ (1977), 198201.

[13] Huet, D., Décomposition Spectrale et Opérateurs, Presses Universitaires de France, (1977).

[14] Kirchhoff, G., Vorlesunger über Mechanik Tauber, Leipzig, 1883.

- [15] Larkin, N.A., Mixed problems for a class of hyperbolic equations, Siberian Math. Journal, 18 (6), (1977), 1414-1419.

[16] Larkin, N.A., Global solvability of boundary value problem for a class of quasilinear hyperbolic equations, Siberian Math. Journal, 22 (1), (1981), 82-88.

[17] Lions, J.L., On Some Questions in Boundary Value Problems of Mathematical Physics, Contemporary Development in Continuum Mechanics and Partial Differential Equations (Ed. by G. de la Penha and L.A. Medeiros), North Holland, London, (1978).

[18] Lions, J.L. and Magenes, E., Problèmes aux Limites Non Homogènes et Applications, Vol. 1, Dunod, Paris (1968).

[19] Maciel, A., On hyperbolic-parabolic equation with a continuous nonlinearity, Nonlinear Analysis, Vol. 20, $\mathrm{N}^{\circ} 6$ (1993), 745-754.

[20] Matos, M.P., Mathematical analysis of the nonlinear model for the vibrations of a string, Nonlinear Analysis, Theory, Methods and Applications, 17 (12), (1991), 1125-1137.

[21] Medeiros, L.A. and Milla, M.M., Solutions for the equation of nonlinear vibrations in Sobolev space of fractionary order, Mat. Aplic. Comp., 6 (1987), 257-276.

[22] Medeiros, L.A., Nonlinear hyperbolic-parabolic partial differential equation, Funkcialaj Ekvacioj, 23 (1978), 151-158. 
[23] Menzala, G.P., On classical solution of a quasilinear hyperbolic equation, Nonlinear Analysis, 3 (1978), 613-627.

[24] Pohozaev, S.I., On a class of quasilinear hyperbolic equation, Math. Sbornic, 96 (1975), 152-156.

[25] Popivanov, N.I., Some boundary value problem for hyperbolic-parabolic equation in multidimensional domains, Soviet Math. Dokl., Vol. 19 (6), (1978), 1390-1394.

[26] Vragov, V.N, , On a mixed problem for a class of hyperbolic-parabolic equation, Soviet Math. Dokl., 16 (5), (1975), 1179.1183.

[27] Yamada, Y., Some nonlinear degenerate wave equation, Nonlinear Analysis, 11 (1987), 1155-1168.

Department of Mathematics

Federal University of Santa Maria

97119-900,.Santa Marỉa,

RS-Brazil

ELENIQBRUFSM.BITNET
Recibido: 9 de Septiembre de 1993

Revisado: 19 de Agosto de 1994 